\title{
Preliminary Conceptual Design of DEMO EC System
}

\author{
S. Garavaglia, ${ }^{1}$ a) W. Bin ${ }^{1}$, A. Bruschi ${ }^{1}$, G. Granucci ${ }^{1}$, G.Grossetti ${ }^{2}$, J.Jelonnek ${ }^{3}$, \\ A. Moro ${ }^{1}$, N. Rispoli ${ }^{1}$, D. Strauss ${ }^{2}$, Q.M. Tran ${ }^{4}$ and T. Franke ${ }^{5,6}$ \\ ${ }^{1}$ Institute of Plasma Physics "P.Caldirola", National Research Council of Italy, Milan, Italy. \\ ${ }^{2} I A M-A W P,{ }^{3} I H M$, KIT, Kaiserstr. 12, D-76131 Karlsruhe, Germany. \\ ${ }^{4}$ CRPP, EPFL, EURATOM Association, CH-1015 Lausanne, Switzerland. \\ ${ }^{5}$ EUROfusion Consortium, Boltzmannstr. 2, D-85748 Garching, Germany. \\ ${ }^{6}$ Max-Planck-Institut für Plasmaphysik, Garching, Germany \\ ${ }^{a)}$ Corresponding author: garavaglia@ifp.cnr.it
}

\begin{abstract}
In the framework of EUROfusion Consortium the Work Package Heating and Current Drive addresses the engineering design and $\mathrm{R} \& \mathrm{D}$ for the electron cyclotron, ion cyclotron and neutral beam systems. This paper reports the activities performed in 2014, focusing on the work done regarding the input for the conceptual design of the EC system, particularly for the gyrotron, the transmission line and the launchers.
\end{abstract}

\section{INTRODUCTION AND PHYSICAL REQUIREMENTS}

In the present European fusion strategy the demonstration fusion reactor (DEMO) is considered as single step between ITER and a commercial Fusion Power Plant. In the framework of EUROfusion Consortium the Work Package Heating and Current Drive addresses the engineering design and the R\&D for the electron cyclotron (EC), ion cyclotron and neutral beam heating systems. The EC system is in charge of key functions as initiating, sustaining and assisting the termination of a tokamak plasma discharge. This means that a very high reliability must be a priority in the concept of the EC system. The identification of EC physical requirements considers two different device concepts, the pulsed EU DEMO1 2015, where the role of current drive (CD) is reduced and EC is mainly dedicated to plasma start-up, ramp up to burn, MHD instabilities control, ramp down, and the steady state EU DEMO2 2015, where significant CD has to be provided in order to sustain the plasma current. EU DEMO1 2015 is the reference design to develop the machine concept. In Table 1 the main parameters of the EU DEMO1 2015 from April 2015 reference design are summarized. The studies for this paper were realized with the former last valid design as it was DEMO1 from July 2012; the Table 1 shows the latest PROCESS CODE results on it for comparison.

The starting point of EC system conceptual design has been the identification of the physical requirements demanded to the EC heating and current drive systems. The main tasks expected to be performed by EC waves are summarized in Table 2 with the required power, deposition localization and frequency. The use of EC to assist the plasma startup is to foresee since DEMO will be a superconducting machine with a limited toroidal electric field as ITER and JT60-SA. The preliminary simulations [1] suggest that a power of 6 to $10 \mathrm{MW}$ is necessary to sustain the DEMO startup and indicate that an oblique injection of the RF power is required to exploit the polarization conversion of the non-absorbed power occurring at inner wall [2]. The METIS [3] simulation conducted in the ramp-up task demonstrated the role of EC power deposited off-axis $(\rho=0.7)$ in keeping low the internal inductance during the current rump and in favoring the $\mathrm{L}-\mathrm{H}$ transition. At present the role of $\mathrm{CD}$ in this phase is not consolidated yet. During the burning phase the EC power is dedicated to Neoclassical Tearing Modes (NTM) and sawtooth control; in addition it can be considered as an option for electron heating in case of fault or unavailability of other systems. The analysis associated to NTM indicated the deposition ranges (see Table 2 ) to control the modes $\mathrm{m} / \mathrm{n}=2 / 1$ and $\mathrm{m} / \mathrm{n}=3 / 2$; the two values refer to different density profiles (flat or peaked) [4]. Some CD close to $\mathrm{q}=1$ 
surface $(\rho=0.25)$ [5] is required to control large sawtooth, dangerous for NTM seeding and consequent possible disruption. A similar table has been realized for DEMO2 2012 design, where the main difference is the required CD necessary for steady state. In this case the EC system will be used to distribute the power along the major radius $(0.2<\rho<0.7)$ in order to obtain a stable current profile [6]. The $q$ profile is expected more flat, therefore no sawtooth or $\mathrm{m} / \mathrm{n}=3 / 2$ instabilities are expected. A total of $50 \mathrm{MW}$ of EC power in the plasma is the reference for the present analysis, assuming also that this power must be guaranteed at maximal availability for all the DEMO pulses.

TABLE 1. Summary of major DEMO1 parameters for the reference design (July 2012) and the latest design (April 2015).

\begin{tabular}{lcc}
\hline & DEMO1 2012 & EU DEMO1 2015 \\
\hline Major radius [m]/minor radius [m]/Aspect Ratio & $9.0 / 2.25 / 4$ & $9.072 / 2.927 / 3.1$ \\
Toroidal field [T] / Plasma current [MA] & $6.8 / 14.0$ & $5.7 / 19.6$ \\
Heating power [MW] / Fusion Power [MW] / $\mathrm{q}_{95}$ & $50 / 1572 / 3$ & $50 / 2037 / 3.247$ \\
$<\mathrm{n}_{\mathrm{e}}>\left[10^{20} \mathrm{~m}^{-3}\right] /$ peaking & $0.9 / 1.5$ & $0.8 / 2.0$ \\
$<\mathrm{T}_{\mathrm{e}}>[\mathrm{keV}] /$ peaking $\left(<\mathrm{T}_{\mathrm{i}}>[\mathrm{keV}] /\right.$ peaking) & $10.5 / 2.5$ & $13.1 / 2.1$ \\
Number of TF coils / Pulse duration [hours] & $16 / 2.29$ & $18 / 2$ \\
Fraction of $\mathrm{I}_{\mathrm{BOOTSTRAP}} / \mathrm{I}_{\mathrm{OHMIC}} / \mathrm{I}_{\mathrm{CD}}$ & $0.361 / 0.635 / 0.004$ & $0.347 / 0.557 / 0.096$ \\
\hline
\end{tabular}

TABLE 2. Main DEMO EC applications. Data refer to DEMO 2012 design, with Aspect Ratio $A=4$. $f_{1}$ depends on toroidal field $B_{o}, f_{2}$ on $B_{o}$, electron temperature $T_{e}$ and launching angle, with $f_{2} / f_{1} \sim 1.2-1.25$ (preliminary estimates with GRAY code)

\begin{tabular}{|c|c|c|c|c|}
\hline & $\begin{array}{c}\text { Power } \\
\text { Required }[\mathrm{MW}]\end{array}$ & $\begin{array}{c}\text { Localization } \\
{[\rho]}\end{array}$ & Mode & Freq. \\
\hline Breakdown and start-up & $6-10$ & 0 & Heating & $\mathrm{f}_{1}$ \\
\hline Ramp up & 50 & 0.7 & $\mathrm{CD}[\mathrm{tbc}]$ & $\mathrm{f}_{2}$ \\
\hline Main Heating & 50 & 0.2 & Heating & $\mathrm{f}_{1}$ \\
\hline $\mathrm{m} / \mathrm{n}=3 / 2(2 / 1)$ Stabilization & 10 & $0.4-0.46(0.6-0.66)$ & $\mathrm{CD}$ & $\mathrm{f}_{2}$ \\
\hline Sawtooth control & $10[\mathrm{tbc}]$ & 0.25 & $\mathrm{CD}$ & $\mathrm{f}_{2}$ \\
\hline Impurity control & 5 & 0 & Heating & $\mathrm{f}_{1}$ \\
\hline Disruption control & 10 & $0.34 / 0.66$ & Heating/CD & $\mathrm{f}_{1} / \mathrm{f}_{2}$ \\
\hline Ramp down & 50 & 0 & Heating & $\mathrm{f}_{1}$ \\
\hline
\end{tabular}

\section{INPUT TO THE CONCEPTUAL DESIGN OF THE GYROTRON}

In the last years gyrotrons have demonstrated high power (1MW for W7-X, ITER), long pulse (1800s for W7-X, 3600 s for ITER) operation in a broad frequency range already (105GHz (AUG), 126GHz (TCV), 140GHz (FTU, W7-X), $170 \mathrm{GHz}$ (ITER) and $240 \mathrm{GHz}$ in research for possible future DEMO ECCD). Hence gyrotrons are able to operate at different magnetic fields of the future DEMO tokamak. The choice of the operating frequency will depend on the selection of the Aspect Ratio (AR) (linked to the toroidal magnetic field) and on the relevance of current drive (CD) and plasma control. The final AR is part of the physical studies in EUROfusion currently. In fact the selected frequency influences the $\mathrm{CD}$ efficiency and the $\rho$ reachable, which is also a function of toroidal launching angles and plasma parameters (e.g. temperature and density). In order to fit all the physical requirements of Table 2 , multi-purpose (multi-frequency) frequency step-tunable gyrotrons are under investigation. The definition of a "multi-purpose operation" shall target the operation of the gyrotron at frequencies which will be multiples of the $\lambda / 2$ wavelength of the RF output window. Assuming a CVD-diamond window and a typical window thickness $(\sim 1.8 \mathrm{~mm})$ will result in frequency steps of about $34 \mathrm{GHz}$. That will allow operation of the gyrotron at different magnetic field configurations (slowly-varying) and at different optimum frequencies for heating and current drive (e. g. 136/170/204/238GHz) [7]. A multi-purpose gyrotron will require a very careful selection of a series of operating modes with very similar caustic radii. First multi-purpose operations have been proposed resp. demonstrated already for W7-X, AUG, TCV and JAEA. Application of ECCD for plasma control in DEMO will require remote steering or fast frequency tuning of gyrotrons, The latter requires a frequency step-tunable gyrotron using a broadband RF output window (preferably Brewster-angle window) and it requires the proper operation of 
the gyrotron at neighbouring modes $(2-3 \mathrm{GHz})$. The principle of frequency step-tunability has been demonstrated for the KIT 1MW D-band multi-frequency short-pulse prototype gyrotron for example [8]. Operating frequencies above $230 \mathrm{GHz}$ will be required for optimum ECCD efficiencies if assuming a high magnetic field at the tokamak as defined for DEMO baseline 2012 [9]. Therefore, the principle feasibilities of a $236 \mathrm{GHz} 1 \mathrm{MW}$ conventional-cavity and, alternatively, a $238 \mathrm{GHz} 2 \mathrm{MW}$ coaxial-cavity gyrotron are currently under investigation at EUROfusion [10]. Beside an output power of $1-2 \mathrm{MW}$, the future gyrotron will require output efficiencies above $60 \%$. It is assumed that the introduction of so-called multi-stage depressed collectors (MSDC) will allow efficiencies above $60 \%$ whereas the target for ITER is $50 \%$ currently. Additionally, a high reliability is also required ( $98 \%$ [tbc], $95 \%$ demonstrated for Russian ITER gyrotron [11]) to guarantee the power delivered to the plasma. An estimate of total number of units required has been done using binomial distribution for the calculation of the probability. For 50MW power delivered to the plasma, considering a $2 \mathrm{MW}$ unit power, $10 \%$ of losses (see next section) and $98 \%$ of reliability of a single gyrotron system, a number of 30-34 gyrotrons are required to ensure $98 \%$ to almost $100 \%$ probability. The reliability of Transmission Line (TL) and Launchers has not included for the moment.

\section{TRANSMISSION LINES}

The main purpose of a generic TL is to transmit the RF power from the sources to the launchers with the highest possible efficiency and mode purity. In addition the TL achieves other tasks as the control the beam polarization, the power monitor, the switch of the beam, the gyrotrons condition, the tritium confinement and to allow the accessibility of each component. Considering the harsh environment of reactor and of the parts directly face on it, a more TL flexibility is desirable to cover functions so far assigned to the launcher and thus to simplify its role.

A target value for the efficiency of TL is $90 \%$ in line with ITER Evacuated Waveguides (EWG) $90 \%-92 \%$ [12] and W7-X Quasi Optical (QO) solution 90\% [13]. For a realistic evaluation of the TL length it is required to know the distance between the torus and the gyrotron hall and the number/positions of the launchers on the tokamak. A power handling in a range from $2 \mathrm{MW}$ to $4 \mathrm{MW}$ allows enough flexibility in the choice of the power per unit: $2 \mathrm{MW}$ (ITER spec. [12]) in the case of a single source for line and 4MW combining two sources in the same line. The same criterion can be used for the Waveguide (WG) internal diameter specification: from $63.5 \mathrm{~mm}$ (ITER, 170GHz, $2 \mathrm{MW}$ [12]) for a single beam per TL to $90-100 \mathrm{~mm}$ for a single beam with reduced losses or two combined beams. Considering the foreseen use of a large number of sources and of several launchers a key role is demanded from inline switches. One or more switch per line is needed to feed different launchers (equatorial and vertical) according to the physical requirements. An alternative solution to the mechanical switches, recently developed also for ITER, is a diplexer-combiner, a compact device for the fast switching of the power between two lines or the combination of two gyrotrons in one single TL [14].

All microwave components of the TL should ideally be compatible with multi-frequency or step-tunable gyrotrons (in particular polarizers, power monitor and matched load), and their layout and organization have to be optimized for simplifying the maintenance accessibility by using remote handling. The control of polarization of the wave has to be considered especially for those components that are sensitive to input polarization, as windows (if with Brewster angle) and diplexers. A CW evacuated matched load compatible with gyrotron multi-frequency is foreseen for gyrotron conditioning after an arc.

For the selection of EC system TL different concepts are considered: a QO transmission line based on several mirrors transmitting the $\mathrm{TEM}_{00}$ mode in air (as in W7-X EC system) and an EWG transmitting $\mathrm{HE}_{11}$ mode (as in ITER, DIII-D, JT-60). The comparison will be based on analysis of (i) advantages/disadvantages of losses, (ii) occupied volume, (iii) required maintenance, (iv) accessibility during operation, (v) auxiliary equipment (cooling, vacuum, safety) and (vi) arc risk.

Also an Evacuated Quasi Optical TL is under evaluation, based on the use of a QO multiple-beam line under vacuum. This solution might be convenient in terms of saved volume and safety with respect to a QO TL in air.

\section{LAUNCHER}

With the tasks foreseen for the DEMO EC system in Table 2 a preliminary concept for the antenna design is ongoing. General guidelines for a conceptual design of the launcher have been identified and options are proposed, counting on two possible accesses: Vertical Port (VP) and Equatorial Port (EP).

The power deposition location (determined by the intersection of the beam with the resonant layer corresponding to the source frequency) in both cases can be tuned either by varying the source frequency or the beam launch angle. 
For frequency tuning (FT) the antenna could consist of a truncated waveguide launching a divergent Gaussian beam with a fixed poloidal and toroidal injection angles, while for angular tuning the candidate option is the Remote Steering (RS) waveguide, which would grant a continuous (limited to approx. $\pm 15^{\circ}$ ) steering range with no movable parts or mirrors in plasma proximity, but recessed out of the port. The option has been extensively studied during ITER ECRH Upper Launcher Design [15] and the implemented in the EC Launcher of the W7-X stellarator [16].

In both RS and FT (multi-frequency/frequency tuning) it still has to be assessed which variant is able to fulfill requirements, taking into account the TL components, launcher internal optics and RF windows compatible with the angular or frequency tunable solutions.

Some constraints on antenna specifications may be relaxed due to the restricted operational parameters in DEMO, for example a reduced launched beam flexibility with respect to ITER case. On the contrary some other requirements may rise (related to long EC pulse or multi-frequency gyrotrons regimes). Although the RS layout could fit into the Equatorial DEMO Port (space restrictions are particularly demanding in the case of VP), the implementation strongly depends on the precise launching angle the antenna will have to aim at, and on the engineering constraints imposed by the different interfaces with DEMO Vacuum Vessel, Blanket and Remote Handling (RH) systems. With major benefits with respect to others DEMO Heating Systems, ECH will require openings into the blankets that have to keep as low as possible bot to degrade the Tritium Breeding Ratio from the target value TBR $>1.1$. Although minimal tritium breeding space occupation would be required either for a RS antenna or a truncated waveguide launcher, a careful choice of the number of openings and their sizes alongside with the breeder material to allow Tritium self-sufficiency must be anyway made [6]. The RS option limits must be also considered: (i) the waveguide length is a few meters and increases with frequency; (ii) the waveguide routing is not straightforward (mitre bends can be inserted only in special position); (iii) the RS waveguides are difficult to pack close to one-another since larger space is needed for the input mirrors; (iv) cooling requirements are much stronger than in a circular waveguide. The challenging integration of the multiple EC tasks in a feasible launcher configuration using either frequency or angular tuning and adapted to engineering and physics requirements is the aim of the future work.

\section{FUTURE PLANS AND ACKNOWLEDGMENTS}

Next steps for 2015, starting from an update of the physical requirements, are: (i) the definition of frequency and flexibility of gyrotrons; (ii) for TL the choice of solution (WG or QO), a first conceptual design, an identification of critical components requiring further development, (iii) the reference design for the short pulse RF load and (iv) for the launcher the type selection and first conceptual design.

This work has been carried out within the framework of the EUROfusion Consortium and has received

funding from the Euratom research and training programme 2014-2018 under grant agreement No 633053. The views and opinions expressed herein do not necessarily reflect those of the European Commission.

\section{REFERENCES}

1. L. Porte, EFDA_D_2LMFTV (2013)

2. G. Granucci et al., $25^{\text {th }}$ IAEA Fusion Energy Conference, San Petersburg, Russia (2014) EX/P2-51

3. J.F. Artaud et al., in $32^{\text {nd }}$ EPS Conf. on Control. Fusion and Plasma Phys., ECA Vol. 29C, P1.035 (2005)

4. R. Wenninger et al., Nucl. Fusion 55 (2015) 063003

5. G. Giruzzi et al., $25^{\text {th }}$ IAEA Fusion Energy Conference, San Petersburg, Russia (2014) TH/P1-14

6. G. Grossetti, $9^{\text {th }}$ workshop on Strong Microwaves \& THz Waves, Nizhny Novgorod, Russia (2014) H-19

7. J. Franck et al, IEEE International Vacuum Electronics Conference (2014) 31-32

8. G. Gantenbein et al., IEEE Transactions on Electron Devices 61, 6, 1806-1811 (2014)

9. E. Poli, Nucl. Fusion 53 (2013) 013011

10. J. Jelonnek, German Microwave Conference (GeMIC) (2014) 1-4

11. G. Denisov, A. Litvak, $25^{\text {th }}$ IAEA Fusion Energy Conference, San Petersburg, Russia (2014)

12. F. Gandini et al. Fusion Sci. Technol. 59, 709-717 (2011)

13. V. Erckmann et al. Fusion Sci. Technol. 52, 291-312 (2006)

14. D. Wagner, W. Kasparek, US-EU-JPN Workshop on RF Heating Tech., Sedona, Arizona, USA (2014)

15. M. Graswinckel et al., Nucl. Fusion 48 (2008) 054015

16. B. Plaum et al., Journal of Physics: Conference Series 25 (2005) 120-129 